Most curious is his endorsement of the view (first stated by Derek Price in 1957) that Copernicus was no revolutionary. It seems odd to minimize the cosmological and epistemological significance of Copernicus; if the Pythagoreans had long preceded him, Newton (with less occasion, we may think) allowed as much with respect to the theory of gravitation, for he thought that the Pythagoreans must have understood this also. No greater tribute could be paid to Copernicus than that implied in Newton's conclusion to the first Scholium of Principia Mathematica (1687): I wrote the following treatise, he declared, with the object of elucidating true motions and causes in nature from apparent causes and effects, and conversely of elucidating causes and effects from both true and apparent motions. If Copernicus had not proposed this problem, who did?

The Newtonian system without Copernicus as the first of the "giants" is indeed inconceivable. Bernard Cohen's difficulty arises from the perhaps inavoidable confusion between the idea of revolution as the work of an individual (Lenin, Lavoisier) and the idea of revolution as the fruition of a movement (bolshevism, pneumatic chemistry). Sometimes Cohen seems virtually to be defending the "great man" concept of change, or even (in his discussion of Wegener) the "neglected genius". More frequently, he seems to mark the great men on a scale of revolutionary activity or fervour: thus Bacon's great contribution to methodology "did not constitute a revolution in science"; Gilbert "did not establish a new science", unlike Galileo, "a heroic figure of the Scientific Revolution", although even "Galileo's revolution was not complete"'. Descartes's revolution "did not last" and "it seems clear there was no Keplerian revolution in science before 1687 '. One remembers that Alfred was a Good King. What is historically important is an understanding of how the separate, even disparate, endeavours of individuals contemporaneous with each other or in quick succession, culminate in some particularly noteworthy and well-founded shift in our vision of nature, for few historians believe that scientific revolutions are literally effected single-handed by a unique scientist. And so to me, for example, it makes good sense to speak of a "revolution in geology" between about 1790 and 1840 , whereas talk of a "Lyellian revolution" would be unjustifiable. Or again, it is more constructive to consider the triumvirate Faraday-Maxwell-Hertz as a movement, than to try to weigh out the praise for innovation due to each.

Like many large historical works, Bernard Cohen's book excels as a quarry, rather than as a coherent argument. $\mathrm{He}$ is likely to have the last word for a long time to come.

A. Rupert Hall is Emeritus Professor of the History of Science and Technology at the University of London.

\section{Agents of change}

\section{S. Venitt}

Chemical Carcinogens, 2nd Edn. ACS

Monograph 182.

Edited by Charles E. Searle.

American Chemical Society: 1984. Pp.

1,373. \$129.95 (North America), \$155.95 (elsewhere).

UNTIL recently, the study of carcinogenesis has been largely correlative and phenomenological. What does chemical A do to biological systems X, Y and Z? Does B do it better and is it metabolized to $C$ ? What happens when you apply A before B, or B before A, or mix A and B together? And so forth. This approach has been very fruitful: we now know, for example, that most carcinogens are genotoxic and that most non-carcinogens are not; that many carcinogens are themselves electrophiles or are metabolized to electrophiles; that some substances "promote"' tumours but do not initiate them; that many carcinogens are organotropic; and that carcinogenesis is a multi-step process. Much of this information was gathered together in the first edition of Chemical Carcinogens, published in 1976.

The study of the mechanism of carcinogenesis was transformed and became at once both analytical and synthetic when two groups independently showed that NIH-3T3 cells could be transformed in vitro by transfection with DNA from a human bladder tumour, and that a single base change within a proto-oncogene was in some cases sufficient to activate the oncogene. There has followed a flood of research (which shows no sign of abating) which stresses the importance of both point mutation and chromosomal rearrangement in the activation of proto-oncogenes. The idea that DNA is the critical target for carcinogens has thus gained support from two different streams: the older, painstaking, sometimes stumbling correlative approach, and the rather more breezy and with-it analytical methods of cell biology and recombinant DNA technology.

Nevertheless, "descriptive chemical carcinogenesis" will not go away. We still need to know whether chemicals, new or already in use, are likely to be carcinogenic. In this light appears the second edition of the American Chemical Society's Chemical Carcinogens, which offers an expanded view of the descriptive approach embodied in the first edition.

ACS Chemical Monographs are intended to serve two main functions: (i) "to make available to chemists a thorough treatment of a selected area in form usable by persons working in more or less unrelated fields to the end that they may correlate their own work with a larger area of physical science"; and (ii) "to stimulate. further research in the specific field treated". The first edition filled these roles ○)1985 Nature Publishing Group admirably, not only for chemists, but also for a wider audience interested in chemical carcinogenesis. This second edition, in two well-indexed and richly-referenced volumes, is a valuable update and expansion: there are now 22 chapters (all by leading specialists in each field) instead of 16 .

Included are completely new chapters on cancer epidemiology, DNA-carcinogenmetabolite interactions, halogenated organic compounds, inorganic carcinogens, environmental $N$-nitroso carcinogens, triazenes, hydrazines, azo and azoxy compounds, aflatoxins, fusarial mycotoxins, carcinogenic medicines, and the inhibition of chemical carcinogenesis. Other topics which were covered in separate contributions in the first edition (for example, metabolism of carcinogens, carcinogens in plants and microorganisms) are now spread over several. Although much of the core of the first edition remains (polynuclear aromatic hydrocarbons; soots, tars and oils; aromatic amines and their epidemiology; laboratory hazards; alkylating agents; mineral fibres; $N$-nitroso compounds; bracken carcinogens; carcinogens in food; bioassays and short-term tests) several important topics are omitted, such as tumour promotion and co-carcinogenesis, endocrine aspects of carcinogenesis and respiratory carcinogenesis. This is a pity, and it is fair to assume that the vagaries of deadlines and non-availability of authors dictated the final content.

Much of the material consists of detailed and very helpful tabulations of carcinogens and descriptions of their metabolism and biological activity, with much emphasis on structure-activity relationships. In a text of this size and scope there are bound to be some contributions which are more thorough or more readable than others and it is tempting to give each of them marks out of ten. Although I have resisted the urge I must nevertheless single out Lawley's contribution on carcinogenesis by alkylating agents as the chapter which gives the deepest and broadest view of chemical carcinogenesis as it stood in 19811982. Viewing alkylating agents as "archetypal carcinogens", he provides us with a masterly, wide-ranging review, which includes discussions of chemical reactivity, dose-response relationships, mechanisms of mutagenicity, short-term tests, DNA repair, models of carcinogenesis, alkylating agents and human cancer and, as a final flourish, squeezes in (in a note added in proof) a short account of the first reports of activation of proto-oncogenes by point mutation.

Orchestrating such an encyclopaedic text must have been an awesome task. The editor, Dr C.E. Searle, must be congratulated for producing such a useful revision of an already indispensable work.

S. Venitt is a member of the Section of Chemical Carcinogenesis, Institute of Cancer Research, Royal Cancer Hospital, Sutton, Surrey. 